\title{
A Novel Music Therapy Intervention Utilizing Pop Music to Reduce Adolescent Anxiety and Depression
}

\author{
Zhixing Huang ${ }^{1}$ and Natasha Duell ${ }^{1}$ \\ ${ }^{1}$ Xiamen HaiCang Vocational School \\ Belmont High School, Belmont, MA, USA
}

\section{ABSTRACT}

The prevalence of adolescents' mental disorders, especially anxiety and depression, has become a critical social issue that needs to be addressed. Conventional music therapy could be a useful tool to help teens manage their symptoms. However, few kinds of research have explored the potential function of listening to pop music to improve adolescents' mental state. This paper explored a pivotal approach to whether scientifically proven pop music can help prevent anxiety and depression. The paper developed a criterion that is used for selecting pop music with mentally beneficial elements and created a playlist with songs selected under the criterion. Two survey-based studies, containing a professional diagnosis scale and various questions, qualitatively and quantitatively examined the playlist's emotional impact. In Study 1, results indicated that the playlist could relieve anxiety symptoms, with less influence in depression. In Study 2, regular control measures and qualitative questions proved that the selected music exerted its intended positive emotional effects onto the listener and indicated that listening to the music on a daily basis may be useful in improving and maintaining one's healthy mental state. The results were promising and suggested great potential in future research. The scientific use of pop music as a self-help method beneficial in tackling anxiety and depression among teenagers could be a prospective field of study.

\section{Introduction}

In recent years, disorders among adolescents have become a critical social issue. Mental disorders, such as depression and anxiety, are among the major causes of disease, disability, and death in the world (World Health Organization, 2019). The impact of adolescents' mental disorders on their families and communities is wide-ranging and long-lasting (Sethi, 2010), including socioeconomic outcomes that continue into adulthood (Knapp, 2002). In recent years, researchers and policymakers have advocated for more investment in developing multi-pronged preventive intervention programs that may help adolescents who are at a high risk of developing a mental disorder or have preclinical symptoms (Thapar \& Collishaw \& Potter, 2010). Among such programs are those emphasizing self-help treatments, including music therapy, to minimize the symptoms of mental disorders in adolescents' daily lives (Morgan \& Jorm, 2008). Findings from several studies have supported the benefits of music to mental health (Lin et al., 2011; Miranda, 2013). Although most music therapy programs require in-person visits with licensed therapists, it may be possible for adolescents to benefit from music in more accessible ways. To this end, the present study tests the effectiveness of a novel music therapy intervention that requires nothing more than access to a music playlist in reducing symptoms of anxiety and depression in adolescents.

Anxiety and depression are two of the most prevalent mental disorders among adolescents. According to Maughan et al. (2012), they are comorbid, meaning they frequently co-occur, and one will increase the risk of the other as time passes. The diagnostic criteria for depression "center on core symptoms of persistent and pervasive sadness, along with a loss of interest or pleasure in activities."(Maughan et al., 2012). Anxiety is characterized by feelings of tension and worrying thoughts, and somatic symptoms like an increase in blood pressure. Depression and anxiety during adolescence are known to interfere with personal relationships and academic performance (Garber \& 
Weersing, 2010). These disorders may also increase risks of suicide and other psychopathologies. These factors can propagate forward into adulthood(e.g., Gould et al., 1998; Rohde et al., 1994; Weissman et al., 1999). Moreover, anxiety and depression go unnoticed in teens, as teenagers are reluctant to let others understand their symptoms. Less than $50 \%$ of teenagers would seek mental health services, and this reluctance may be attributed to the unawareness of symptoms, financial hurdles, and psychological barriers (Sethi, 2010). In light of the above evidence, the importance of developing an effective and highly accessible technique for helping adolescents manage their symptoms goes without saying.

Music therapy may be a useful tool to assist young people in managing their symptoms of anxiety and depression. Several studies have shown music to be an effective therapeutic technique in treating depression and anxiety (Lin et al., 2011; Miranda, 2013). Music Therapy is consists of two fundamental methods: the "receptive", listeningbased method versus the "active" method of creating sounds through playing music with instruments or composing (Guetin et al., 2009). The "receptive" method, which is the focus of this study, is described as a "controlled method for listening to music, making use of its physiological, psychological and emotional impact on the individual during treatment for an illness or trauma"(Biley, 1992). Systematic reviews of music interventions have discovered evidence in the effectiveness of music in treating symptoms of depression and anxiety. For example, in Goldbeck and Ellerkamp (2012), music therapy is accessible to youth who struggle to verbalize their feelings by targeting the expression and regulation of emotions without requiring them to communicate their feelings verbally. Additionally, this research shows that relaxing music has been shown to help anxious teenagers regulate their stress levels. Another unique benefit of using music to help manage depression and anxiety is that it is highly accessible as a self-help tool for teens. The supply of preventive interventions lags far behind the rising rates of adolescent mental problems due to a severe worldwide shortage of professional psychiatrists and a trained workforce(Skokauskas et al., 2019). As a result, researchers and policymakers have advocated for programs emphasizing on using self-help treatment to prevent and reduce the symptoms of mental disorders in daily life(Morgan \& Jorm, 2008). Therefore, an accessible music therapy intervention may be a promising way to help teens manage their symptoms in their everyday life.

One issue with current music therapies is that the "relaxing" and "healing" music used in these therapies are often classical or instrumental music that may be less appealing to teenagers. Existing music therapy programs mainly use classical and instrumental music repertoires because the effectiveness of these music styles in adults has been well-documented. Classical and instrumental music's musical characteristics are also relatively easy to examine (Grocke \& Wigram, 2007). However, the use of more modern music may increase adolescents' investment, interest, and compliance with music therapy programs. $64 \%$ of the people throughout the world listen to pop music; the most listened to genre in the world (IFPI, 2018). Recent reports have shown that popular music was the genre with the greatest number of recommended songs by music therapists to implement in singing activities (VanWeelden \& Cevasco, 2017). Therefore, pop music may be promising for interventions targeted at young people. The use of pop music may also increase adolescents' compliance with therapy programs. However, more substantial research should be done to investigate the effects of pop music on reducing depression and anxiety symptoms in adolescents. More research that adopts well-designed controls of high methodological quality is needed for further development(Geipel $\&$ Koenig \& Hillecke \& Resch \& Kaess, 2018). Future studies should also take into account empirically examine the effectiveness of pop music on adolescent mental health.

Additionally, vast music consumption among teenagers in the 21 st century establishes a foundation for implementing music as a therapeutic technique for managing anxiety and depression. Conventionally, music therapy takes place in a clinical setting and involves various musical tools and the assistance of a licensed professional. However, if music therapy were combined with mobile applications, the therapeutic methods could be implemented from anywhere. Therefore, reimagining music therapy by combining it with technology can result in a cost-effective and easily implementable therapeutic technique, which may dramatically increase adolescents' compliance with their treatment. These music therapeutic methods integrated with technology can be targeted towards a more significant number of teenage audiences and could be widely administered. 
This study investigates the effectiveness of a novel music therapy intervention on reducing symptoms of anxiety and depression in adolescents and answers whether pop music can help reduce adolescents' anxiety and depression. Prior to the two studies, a criterion was developed based on previous research to select pop music with mentally relaxing characteristics. After compiling a repertoire of pop music with relaxing qualities, a playlist was created on the Chinese music app QQ Music. In both studies, participants reported their depression and anxiety symptoms before and after the intervention through the Hospital Anxiety and Depression Scale (HADS). In the first study, a sample of 50 Chinese high school students participated in a week-long experiment in which they listened to the relaxing playlist. The hypothesis for Study 1 is that the participants would show general relieving signs of symptoms of depression and anxiety after listening to the therapeutic playlist every day for one week, with lower scores on the HADS scale. In the second study, a structured group and an unstructured group participated in an eight-day experiment. Their hypothesis for Study 2 is that the participant's emotional responses would conform to the playlist's intended impacts, and listening to the music on a daily basis would be more effective in maintaining a continually healthy mental state.

\section{General Method}

\section{Overview}

In the studies reported here, we examine the effects of a novel music therapy intervention on anxiety and depression scores in adolescents from Mainland China. The first study aimed to determine if the criterion was effective in selecting suitable pop music and if the chosen pop music in the playlist could reduce anxiety and depression symptoms. The second study aimed to explore (1) whether listeners are receiving the intended positive emotional impact; and (2) if regularly listening to the playlist on a daily basis could further improve and maintain the listener's positive emotions.

\section{Music Selection}

A music playlist with 100 pop songs was created using the QQ Music app for the adolescents participating in this study. The QQ Music app is one of the most popular online music streaming services in China that can be downloaded for free on a mobile phone. All participants received the same playlist and could choose to listen to any songs on that playlist in any order and frequency. The playlist was restricted only to popular music, which is defined as "instant singles-based music aimed at teenagers" (Frith, Straw, \& Street, 2007). Pop songs were chosen specifically because of their appeal, enjoyment, and familiarity with teenage audiences.

The pop music selection criterion was designed based on previous research on musical elements most conducive to relaxation (Elliot et al., 2011; Huang, 2011; Lee-Harris \& Timmers, 2018; Saarikallio, 2015; Wang, 2014). We used the criterion created by Elliot et al. (2011) as the foundation to guide our selection of songs for the current study. As the criterion did not take into much consideration of pop music genres and repertoire, certain musical elements from the original criterion were modified and improved to adapt to the characteristics of pop music in our established criterion, including melody, rhythm, instrumentation. The musical component "lyrics" was also added. It did not exist in the original study but had a significant influence on one's emotions in pop music (e.g., Brattico et al., 2011; Mast \& McAndrew, 2011; Besson et al., 2011). The modifications can be found in Appendix B. The final selection criteria are listed in Table 1. To find songs to include in the playlist, we searched Chinese songs with tags such as "stress relieve" and "relaxing" on QQ Music and Spotify and selected the final playlist of songs based on the selection criteria. 
Table 1. Pop Music Selection Criterion: Characteristics of the Internal Components of Mentally Beneficial Pop Music

\begin{tabular}{|l|l|}
\hline 1. Tempo & Not too slow, 80-100 beats per minutes \\
\hline 2. Melody & Strong, obvious and secure, "easy to hum to" \\
\hline 3. Beat & $\begin{array}{l}\text { Constant, regular pose with 4/4 signature,4/3 are less re- } \\
\text { laxing }\end{array}$ \\
\hline 4. Harmony & $\begin{array}{l}\text { Utilize tonal progressions that are popular in Western } \\
\text { music. Less dissonance }\end{array}$ \\
\hline 5. Lyrics & Optimistic words \\
\hline 6. Key & Major keys recommended \\
\hline 7. Articulation & Smooth and connected, less staccato melodies preferred \\
\hline 8. Dynamics & $\begin{array}{l}\text { Relatively constant volume throughout the song. Does } \\
\text { not add on intensity to the participant }\end{array}$ \\
\hline & $\begin{array}{l}\text { Preferably piano and string if appeared in songs. Elec- } \\
\text { tronic components/voice should not sound harsh or loud } \\
\text { to the ears. }\end{array}$ \\
\hline 9. Instrumentation & "Tranquil" character preferred \\
\hline 10. Overall music character and sounding to ears &
\end{tabular}

\section{Study 1}

Study 1 aimed to investigate the possible effects of pop music on relieving and reducing depression and anxiety symptoms. The study evaluated the effectiveness of the novel mentally beneficial pop music selection criterion in selecting appropriate music. It determined whether the selected music from the playlist could help reduce symptoms of anxiety and depression among the participants.

\section{Methods}

\section{Participants}

Fifty juniors and ten seniors from one high school in Xiamen, China, were invited to participate in the first experiment. They aged from 16-18 on average, and the number of male and female participants was both 30 . Twelve surveys were excluded from the results, as their survey responses contained various flaws such as missing answers or choosing the same answer for all questions. The school administration was informed of the experiment objectives and process. One teacher provided consent for the class of students to participate in the study. All participants consented to participate and allow us to use their responses for research purposes.

\section{Measures and Procedures}

Before the music intervention, the participants were asked to complete a demographic questionnaire and the Hospital Anxiety and Depression (HADS) scale (Zigmond \& Snaith, 1983). The HADS scale is a 14-item scale commonly used by doctors to determine the levels of anxiety (e.g., worrying thoughts go through my mind) and depression (e.g., I have lost interest in my appearance) that a person is experiencing(Zigmond \& Snaith, 1983). The scale was chosen because the questions were suitable for teenagers to answer with simple, easy-to-understand language compared to other surveys. The Chinese version of the HADS scale was referenced from Ye \& Xu (1993), and made into an online survey through DiaoChaXing, equivalent to Google Forms in China. 
Participants answered each item on a four-point (0-3) scale. Scores for anxiety and depression were calculated separately as a sum score of all responses with possible scores ranging from 0 to 21 for anxiety and 0 to 21 for depression. Scores ranging from 0 to 7 for either subscale could be considered as being in the normal range; a score of 11 or higher is 'Abnormal', indicating the possibility of a mood disorder; and a score of 8 to 10 is 'Borderline Abnormal'. The pre-intervention questionnaires were distributed through DiaoChaXing.

After completing the questionnaire, participants began the intervention. Participants were instructed to listen to the playlist in the track library for at least 30 minutes every day. Previous research suggests that 30 minutes of listening time is essential for showing any effects of music intervention (Chan \& Wong \& Thayala, 2011). The participants must select music from the playlist, but the participants could determine the frequency, order, and setting of listening to the music. In order to keep track of the music that students listen to and ensure that the participants are following the rules to the experiment, we randomly selected ten students every day to ask them to send a screenshot of the songs they heard from their listening history to us, which is available on the QQ Music app. All of the ten students asked to send screenshots complied.

After listening to music for one week, the participants took a new survey that contained the HADS scale test.

\section{Results}

Table 2. The distribution of anxiety and depression levels among participants in the pre-test and a week after the pretest

\begin{tabular}{|c|c|c|c|}
\hline \multirow{2}{*}{ Category } & Level/Scoring & Percentage(\%) \\
\cline { 2 - 4 } & & Pre-test & Test after one week \\
\hline \multirow{3}{*}{ Anxiety Level } & Normal (0-7) & 50 & 56.25 \\
\cline { 2 - 4 } & Borderline Abnormal (8-10) & 41.67 & 35.42 \\
\cline { 2 - 4 } & Abnormal (11-21) & 8.33 & 8.33 \\
\hline \multirow{3}{*}{ Depression Level } & Normal (0-7) & 72.92 & 66.67 \\
\cline { 2 - 4 } & Borderline Abnormal (8-10) & 16.67 & 6.25 \\
\cline { 2 - 4 } & Abnormal (11-21) & 10.42 & \\
\hline Total Number of Participants: 48 & & \\
\hline
\end{tabular}

For descriptive purposes, we organized people's depression and anxiety scores into three groups from the HADS scale and conducted paired sample t-tests. Forty-eight sets of valid surveys were received. Results from the pre-intervention HADS questionnaire indicated that $49 \%$ of the participants had "Borderline Abnormal" or "Abnormal" signs of anxiety, and 26\% of the participants had "Borderline Abnormal" or "Abnormal" signs of depression. The distribution of HADS scores for the anxiety and depression subscales and their percentage changes are recorded in Table 2.

Table 3. T-test for HADS total score, anxiety score and depression score before and after listening to the playlist for one week

\begin{tabular}{|c|c|c|c|c|}
\hline \multirow{2}{*}{ Variable } & \multicolumn{2}{|c|}{ M (SD) } & t & P \\
\cline { 2 - 4 } & Before & After & 2.064 & $0.045^{*}$ \\
\hline HADS Total Score & $13.85(4.18)$ & $12.56 \square 4.86 \square$ & 2.115 & $0.040^{*}$ \\
\hline Anxiety Score & $7.58(2.39)$ & $6.90(2.67)$ & 1.231 & 0.224 \\
\hline Depression Score & $6.27(3.16)$ & $5.67(3.06)$ & & \multirow{2}{*}{} \\
\hline
\end{tabular}

$* \mathrm{P}<0.05 * * \mathrm{P}<0.01$ 
To test for changes in anxiety and depression after the music therapy intervention, we conducted paired samples $t$ tests for the HADS total score and the anxiety and depression scores separately. Results suggested the total HADS score, anxiety scores, and depression scores after the 1-week music therapy intervention all decreased, but only the decreasing of the total HADS score and anxiety scores were statistically significant $(\mathrm{p}<0.01)$.

Table 4. Correlation Analysis between the change of HADS scoring and the length of music listening

\begin{tabular}{|c|c|c|c|}
\hline & Change of total scoring & $\begin{array}{c}\text { Change of anxiety scor- } \\
\text { ing }\end{array}$ & $\begin{array}{c}\text { Change of depression } \\
\text { scoring }\end{array}$ \\
\hline $\begin{array}{c}\text { Length of daily music lis- } \\
\text { tening }\end{array}$ & -0.131 & 0.18 & $-0.287 * *$ \\
\hline
\end{tabular}

$* \mathrm{P}<0.05 * * \mathrm{P}<0.01$

Note: $\Delta$ scoring $=$ scoring after one week - scoring of pre-test

A correlation analysis was also conducted to evaluate the relationship between the change in HADS scores, calculated as difference scores, and the length of music listening. The results showed that the depression change score was significantly negatively correlated with the length of music listening, which means that longer listening times were associated with greater declines in depression scores. However, change scores for the total HADS scoring and anxiety subscores were not significantly related to the length of music listening.

\section{Discussion}

Study 1 provided great insight into the teenage participants' current mental state, the effectiveness of the criterion and the selected playlist, and the participant's overall emotional response to the playlist on a preliminary level. The distribution of anxiety and depression levels indicated that the proportion of participants with anxiety and depression is relatively high, with participants with more anxiety symptoms than depression, suggesting that anxiety and depression symptoms are indeed relatively prevalent among teenagers. However, after a week of music listening, the HADS results show an overall decreasing trend, indicating the potential effectiveness of the selective criterion and the playlist. The correlation analysis also suggests a connection between the reduction of anxiety and depression symptoms with the length of music listening throughout the week.

\section{Study 2}

Our first study was successful in terms of how it created a criterion that could be used to select music with relaxing characteristics. In order to specifically evaluate the participant's emotional response with more accuracy and ensure that the participants are receiving the intended positive emotional impact from this pop music, we conducted a second study. The second study went through a duration of eight days, including the preparation time due to time differences in China and the U.S., and the actual experiment length. The study included an interventional and non-interventional group. Compared to the first study, this study specifically evaluated the listener's emotional assessment of the playlist music to ensure that they are receiving the intended positive psychological impact, while also providing more insight into our direction of music selection. We also added a regular intervention to ensure that people take their daily "dose" of music regularly. We hoped to discover if regular and day-to-day control of their listening activities could further maintain their positive emotions.

Methods 


\section{Participants}

The 48 participants that submitted valid surveys in Study 1 continued to participate in Study 2. The participants were randomly assigned into two groups: the structured group $(n=27)$ and the unstructured group $(n=21)$. The structured group was chosen based on stratified random sampling. Stratified random sampling ensured that the structured group participants were from all three levels of 'Normal' 'Borderline Normal' and 'Abnormal' from the HADS scale. Among the 27 people, six people were excluded during the experiment because some of them missed the deadline to submit their daily check-ins during a certain day in the middle of the week. All students consented to participate and allowed us to collect their Wechat information.

\section{Measures and Procedures}

On the first day, all participants were asked to complete a questionnaire and the Hospital Anxiety and Depression (HADS) scale (Zigmond \& Snaith, 1983) that was the same given as Study 1. Starting from day 2, the structured group and unstructured group followed separate protocols.

The unstructured group of students was simply given the same instructions as that of in Study 1. We recommended that they listen to music for 30 minutes, but they were not asked to submit check-ins or daily surveys. The unstructured group was given the HADS scale on the first and the last day of the intervention.

The structured group participants were added into a Wechat group, where they were reminded to listen to music and completed daily surveys to various questions about their emotions. Students were required to listen to music for at least 30 minutes a day for eight consecutive days and were asked to provide screenshots of their listening activity from the QQ Music app user page for confirmation. Students also received a daily survey that included multiplechoice questions that were asked in Chinese. Participants received different surveys across the eight days of the intervention (see Appendix A for the full list of question items organized by day). The surveys included questions that asked specific questions on their emotional response to the music and general questions on their mental well-being. The questions on their emotional reactions to the music mainly included two types. One group questions were designed to understand their specific emotional responses to the music from the playlist. The other group of questions were intended to ask them to evaluate how listening to the playlist impacted their overall mood. They were required to fill out the survey before 11:59 PM daily.

Because all the participants were Chinese, we selected ten adjectives total (five contrasting groups) describing emotional responses. The ten adjectives were originally from the research conducted by Zhong \& Qian (2005). The five contrasting groups were referenced from the study of Cai et al. (2007) - they divided the ten adjectives into five groups, each with two antonyms (e.g., sad and joyful).

On the eighth day of the experiment, the structured group was given the HADS scale and a couple of other questions assessing their overall listening experience. These questions can be found in appendix A.

\section{Results}

Results are reported separately for the structured and unstructured groups.

\section{Quantitative Results for Structured Group}

Table 5. T-test for structured groups's HADS score before and after listening to music for eight days

\begin{tabular}{|c|c|c|c|c|c|}
\hline \multirow{2}{*}{ Variable } & \multicolumn{2}{|c|}{ M (SD) } & \multirow{2}{*}{ Difference } & $\mathrm{t}$ & $\mathrm{p}$ \\
\cline { 2 - 5 } & Before & After & & \\
\hline \multirow{2}{*}{ HADS Total Score } & $14.07(4.42)$ & $12.11(5.47 \square$ & -1.96 & 1.852 & 0.075 \\
\hline
\end{tabular}




\begin{tabular}{|c|c|c|c|c|c|}
\cline { 3 - 5 } Anxiety Score & $7.74(2.28)$ & $5.89(2.62)$ & -1.85 & 2.925 & $0.007^{*}$ \\
\hline Depression Score & $6.33(3.66)$ & $6.22(3.47)$ & -0.11 & 0.141 & 0.889 \\
\hline
\end{tabular}

$* \mathrm{P}<0.05 \quad * * \mathrm{P}<0.01$

The HADS scores were organized in the same fashion as study 1. Results from the structured group's paired sample t-tests showed that anxiety scores significantly decreased after the 8-day intervention. However, there were no significant changes in depression scores. Compared to depression, the playlist may have been more effective in preventing the escalation of anxiety or releasing anxiety symptoms.

\section{Quantitative Results for Unstructured Group}

Table 6. T-test for unstructured groups's HADS score before and after listening to music for eight days

\begin{tabular}{|c|c|c|c|c|c|}
\hline \multirow{2}{*}{ Variable } & \multicolumn{2}{|c|}{ M (SD) } & \multirow{2}{*}{ Difference } & $\mathbf{t}$ & $\mathbf{p}$ \\
\cline { 2 - 3 } & Before & After & -1.47 & 1.609 & 0.125 \\
\hline $\begin{array}{c}\text { HADS Total } \\
\text { Score }\end{array}$ & $12.74(3.97)$ & $11.26(3.87)$ & -1.11 & 1.9 & 0.074 \\
\hline Anxiety Score & $6.95(2.27)$ & $5.84(2.27)$ & -0.37 & 0.552 & 0.588 \\
\hline $\begin{array}{c}\text { Depression } \\
\text { Score }\end{array}$ & $5.79(2.70)$ & $5.42(2.09)$ & & \\
\hline
\end{tabular}

$* \mathrm{P}<0.05 \quad * * \mathrm{P}<0.01$

Compared to the structured group, the unstructured group also showed a decrease in all three mean scores after eight days without interference. However, there were no significant statistical changes among any of the scores. Overall, even without intervention on a daily basis, continual listening to the playlist music may also help to maintain a positive mental state and prevent escalating symptoms of anxiety and depression.

\section{Qualitative Results for Structured Group}

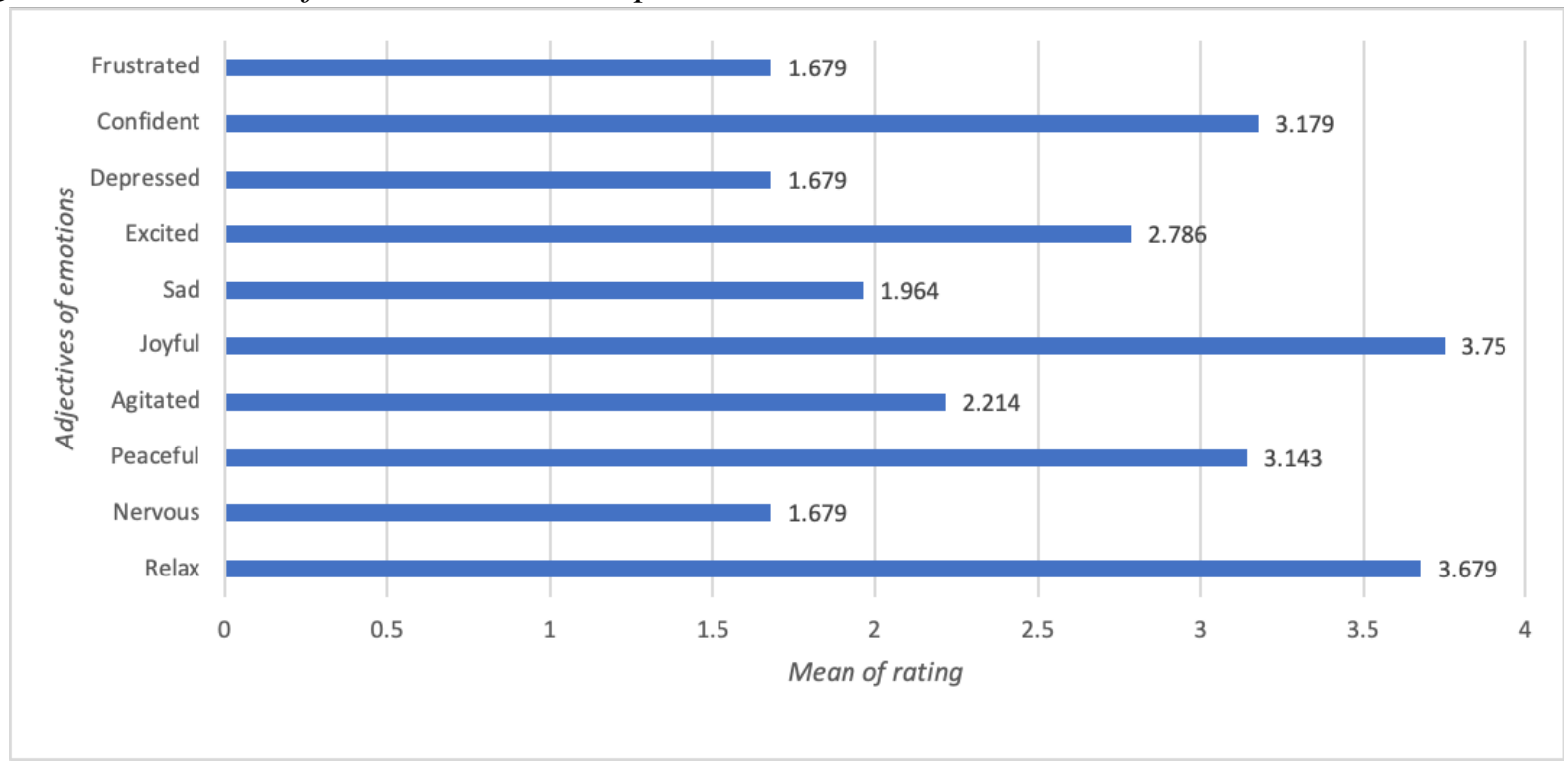


Figure 1. Mean number of the participants' ratings of each adjective as their emotional reaction when listening to the playlist

A variety of qualitative questions were asked in the second study through daily surveys. For the purposes of this study, we chose to focus on questions asking participants to report their emotional feelings using adjectives and rate how much better they felt after listening to the playlist. Figure 1 summarizes the percentage of participants who endorsed various adjectives that reflected their emotional reactions when listening to the playlist. The horizontal axis shows numbers that indicate the participant's emotional response in correspondence to the words on the vertical axis. The emotional reaction is rated on a 0 to 4 scale, with 0 meaning no connection, 4 meaning the closest relationship.

Among the five contrasting sets of words given, all the "positive" adjectives received significantly higher assessment scores than the "negative" adjectives. Among the "positive" adjectives, the participants rated "Happy", "Relaxing", and "Confident" with the highest scores as their most direct reaction to the songs. The statistics supported that the pop music chosen based on the selective criteria conforms to people's emotional response.

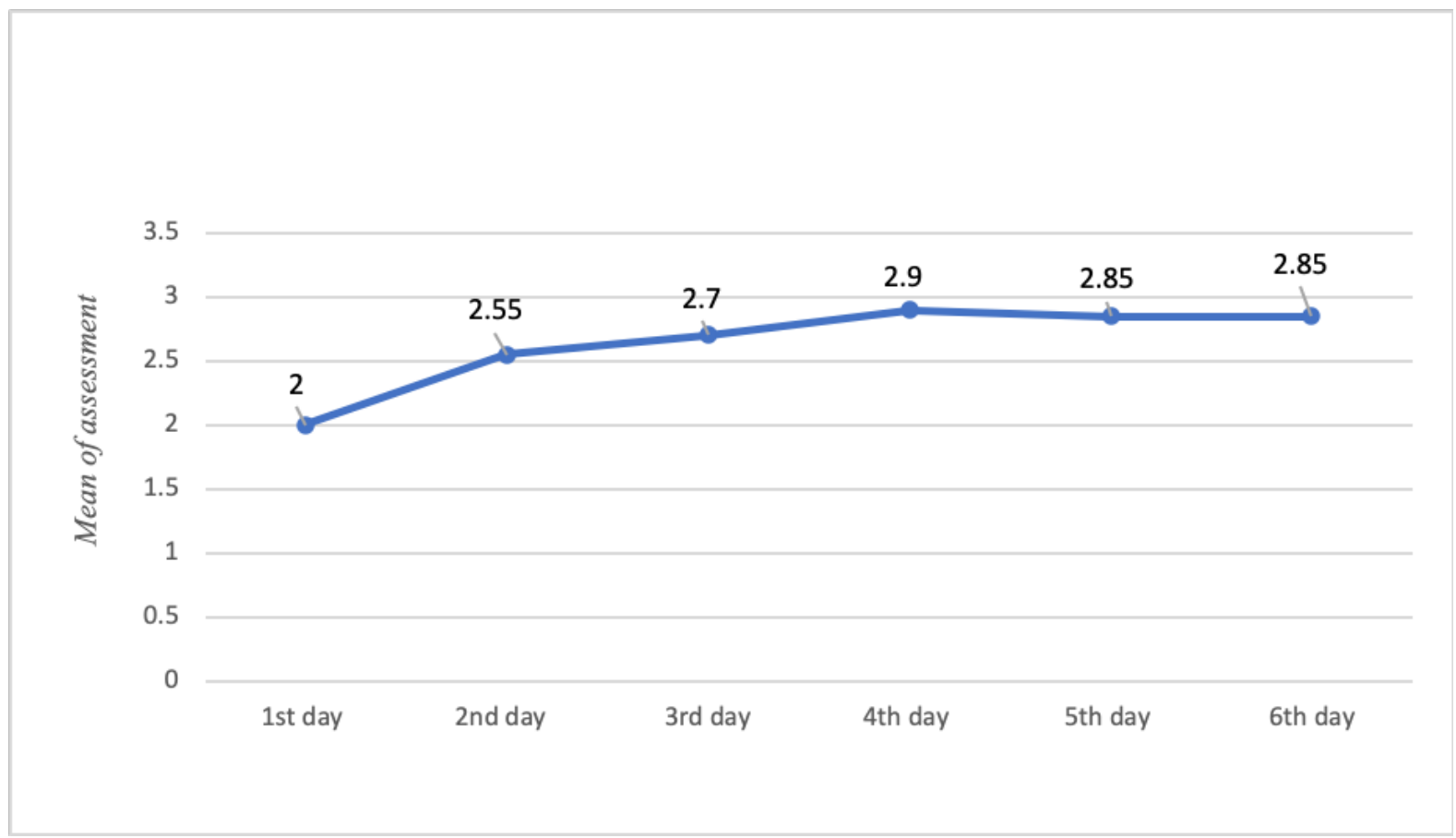

Figure 2. Self-assessment on how the playlist maintained the participants' positive mental state Note. Scores ranged from having no influence (0) to having the most influence(4).

Figure 2 displays the mean rating of participants' assessments of the music playlist on improving their mood each day during the six-day listening period. Scores ranged from having no effect (0) to having the most positive effects(4). Participants' perceptions of the playlist's effectiveness increased during the first four days and maintained a mean of 2.85 since the 5th day. Overall, the participants assessed the playlist as effective in improving their mental state. The upward trend indicates that there could be a correlation between listening to music on a regular daily basis and improving emotional state.

\section{Discussion}

The quantitative results suggested that even though interventive methods were implemented, listening to the playlist for a certain amount of time each day does help prevent or reduce anxiety symptoms, but not necessarily depression 
symptoms. This is similar to the results in the first study in the way that the structured group intervention was more efficient than the unstructured group. At the same time, this data needed to be viewed skeptically because other uncontrollable factors in the participants' personal lives may also impact their mood.

However, another success of this study was reflected in the qualitative results-the intended positive emotional responses from the playlist aligned with the participant 's choice of emotion adjectives. The participants' subjective ratings on "positive" adjectives were much higher than "negative" ones. The participants also regarded the playlist as having improved and maintained their mental state and exerted an overall positive influence on them through their self-assessment scores.

\section{General Discussion}

This study investigated the efficacy of using pop music as a therapeutic tool for reducing anxiety and depression in adolescents. The results supported the hypotheses and provided evidence that listening to a carefully curated pop music playlist could be used as a self-help method for reducing anxiety in teens.

\section{Effectiveness in Relieving Anxiety}

Consistent with previous research showing that music can be relaxing for teens with anxiety (Goldbeck \& Ellerkamp, 2012), results from study 1 showed that pop music intervention reduced anxiety in teens. There are several reasons why this intervention was effective. For one, the act of listening to music can be inherently relaxing. When a person experiences anxiety, they often exhibit tensioned feelings and pressure, and negative thoughts start to emerge (Garber $\&$ Weersing, 2010). However, the songs used in this study were selected specifically for their relaxing musical characteristics and because they contained positive lyrics with uplifting messages. Although not tested directly in this study, hearing relaxing songs with positive messages may reduce stress and counteract negative thoughts. This also reflects the importance of including lyrics in the selection criterion, as words in pop music convey significant meanings compared to instrumental or classical music.

\section{Exception for Depressive Symptoms}

However, it was surprising to find that the intervention was not useful for reducing symptoms of depression. We attribute such differences to the difference in unique aspects of anxiety and depression. According to the tripartite model, anxiety, and depression share characteristics of the common underlying risk factor: general negative affectivity (NA) or negative emotionality (Barlow, 2000; Clark \& Watson, 1991). Garber and Weersing(2010) described NA as "the extent to which an individual feels distressed (e.g., upset, sad, angry, guilty, worried) and not calm, relaxed, or peaceful." However, a negative mood and loss of interest or pleasure in regular activities are unique characteristics of depression, while physical tension and physiological hyperarousal $(\mathrm{PH})$ are distinct features of anxiety. Thus, we predicted that music addressed such "worked-up" aspects of tension and arousal associated with anxiety but was not effective enough to combat the low positive effect and the loss of pleasure and interest associated with depression. Compared to anxiety, depression indicates more signs of hopelessness, worthlessness, and more deep-rooted problems. Therefore, merely using listening-based music therapy to combat depression is not enough. Despite such differences, the statistical significance indicated in the decrease of total HADS scoring and anxiety scoring still shows that the playlist has a general positive influence on the listeners.

\section{Significance of the Selection Criterion}


Compared to "relaxing" songs and playlists recommended by music streaming service providers such as Spotify and QQ Music, the playlist developed for this study was guided by our specially designed empirically-based criterion that specified what musical characteristics would be most effective for therapeutic treatment. In study 2 , the participant's overall self-assessment scoring on the playlist and their high ratings of the "positive" emotional adjectives after listening to the playlist indicated that the music selection was going towards the intended direction. The study also suggested that pop music intervention was indeed effective for minimizing symptoms of anxiety. The establishment of the novel criterion for selecting pop music is very significant. Without the standard to assess the supposedly relaxing or soothing music categories, the effect of such music listening on one's mental health cannot be guaranteed or can even become harmful. Using an empirically-supported standard will help ensure the effectiveness of the intervention. It will also help standardize the treatment across teens, which could be helpful for broader dissemination of the treatment and testing its efficacy in different groups of adolescents. Prospective future research can be directed towards translating the criterion into computer language and applying it for quicker filtering of appropriate pop music, so the playlist could continue to be enriched.

\section{Effects of Regular Control}

In study 2, the interventive methods added for the structured group indicate certain benefits of listening to the selected music daily. If one considers the listening activity as a dose of a new medication that one needs to take, then this study aims to determine whether this 30-minute dose of it is the most effective to maintain emotional stability and improve one's mood. As shown in the results, regular control and daily check-ins could help effectively maintain a healthy mental state and utilize the playlist's function to a greater extent. This establishes an excellent foundation for future research - to promote playlists in the future and keep listeners engaged continually, it is essential to expand the current repertoire and include more songs with different styles and languages.

\section{Practical Significance}

The playlist's approach to music therapy puts the power of the treatment in the hands of teens, enabling them to manage their symptoms independently. This self-help approach is especially crucial for teens concerned about facing mental health stigmas from their parents or peers. Compared to conventional psychiatric services, the playlist is also widely accessible to adolescents as it is an economically viable and easily approachable method for adolescents.

The research on pop music in this study is also unprecedented. As pop music has not been prevalently used for music therapeutic purposes, this study has provided a novel approach to utilizing a more engaging and familiar genre for music therapeutic purposes. Pop music can be seen as a more engaging music genre for listeners, and also broadens the range of music that therapists can use. It increases the possibilities of new research and therapeutic efficacy.

One exceptionally notable fact is that the experiment was conducted during the coronavirus outbreak in China. The participants, like all other Chinese students, are required to be quarantined at home. With sudden academic uncertainty and family changes, as well as the social limitations of being quarantined, many juniors and seniors that face the competition of college entrance exams are increasingly anxious about their lives. Lots of media coverage and news reports had warned the teenage group to be aware of emerging mental issues in such difficult times. Under this context, the data collected from the research during this particular period of time could highlight the benefits of listening to the selected pop music from the playlist and indicate the potential of spreading the playlist to more high school students in need of relief and relaxation during times of uncertainty. 


\section{Conclusion}

The results of this study were encouraging and acknowledge the need for further research on music therapy interventions for teenagers with anxiety and depression disorders. The unprecedented pop music selection criterion established in the study provided more insight into selecting and filtering pop music for therapeutic purposes. Further empirical research in laboratory environments is needed to truly create an effective and mature self-help method through music to prevent anxiety and depression through taking in a "daily dose" of selected pop songs.

\section{Limitations}

\section{Limitations of Study 1}

Due to the study's limitations in the first phase, it is also essential to address the results with more caution. Firstly, despite the study assessing depression and anxiety scores before and after the intervention, there was no control group. The absence of a control group limits our ability to conclude that the changes in the participants' depression and anxiety scores were due to the playlist. Another critical factor that we did not specify was the timing of listening throughout the day. We did not regulate when and on what occasion the participants should listen to the playlist. Therefore, an essential and critical step for future research would be to explore whether the timing of listening to music affects the effectiveness of the treatment. For example, maybe relaxing music right after school can help teens decompress from the day. Or for teens who struggle to sleep due to anxiety, maybe relaxing music would be most useful to listen to before bed.

Another limitation of Study 1 that needs to be considered is whether all the participants listened to the music in accordance with our recommended strategy. Although 10 of the participants were randomly selected to send screenshots through Wechat every day, we did not implement measures to track every participant's adherence to the listening protocol. Therefore, we do not have information about how many teens listened to the therapeutic playlist for thirty minutes every day as they were instructed, which may have created a bias in our results. The study was also implemented during the end of the student's first semester in China, when they have other commitments in their life, such as final exams. This results in a constraint in the study's time length, which could not indicate long-term impacts. We improved this aspect in Study 2 by asking participants to send daily screenshots into a Wechat group chat. Their daily progress could be tracked, and their daily listening activity could be ensured.

\section{Limitations of Study 2}

It is important to note that at its current stage, there is no evidence to suggest that the playlist used in this intervention is effective for treating or preventing anxiety and depression on its own. It should not be considered a replacement for traditional therapeutic techniques. Research has suggested that music therapy, in addition to other psychological treatments such as talk therapy, is often a more effective combination in combating anxiety and depression (Aalbers et al., 2017). Nevertheless, music therapy as an intervention is still essential as it can effectively help prevent the emergence of symptoms and show signs of reducing anxiety and depression in this experiment. It has great prospective research potential that could be further investigated in the future.

At the current stage of this project, the effects of the criterion selected in pop music are still being evaluated based on self-report and response through commonly used medical surveys and were not objective enough. Although subjective responses of emotional adjectives indicate improvement among the participants' mental state, there are several individual difference factors worth considering in future research. For example, compared to a particular student who experienced a substantial academic failure during that week, a student that was not given an assessment and lived his or her regular routine might not feel that much of an impact that the songs have compared to the first student. 
The effectiveness of music therapy differs depending on life experiences; in the future, it will be necessary for researchers to explore how some of those experiences influence the effectiveness of the intervention. Therefore, the study should be improved empirically, considering other factors that can affect peoples' anxiety and their responsiveness to music therapy. Future research could also be strengthened by including physiological measurements through the participant's heart rate or blood pressure or imaging technology such as fMRI to capture brain activity.

\section{Acknowledgments}

I would like to express my deepest gratitude to Wen Huang and her students from the Xiamen Haicang Vocational School for their active participation in these studies that provided such valuable data. The research behind this paper would not have been possible without their exceptional support.

I would also like to sincerely thank Dr. Natasha Duell (the University of North Carolina at Chapel Hill), my supervisor, for her professional guidance. Her patience, supportive encouragement and constructive comments on the research work have greatly improved the paper. Her enthusiasm and knowledge have been a huge inspiration.

I would also like to show my gratitude to Mary Jiang, Katherine Lu, and Shuaiyu Yao for useful discussions and sharing their insightful thoughts with me during the course of this research.

\section{References}

Aalbers, S., Fusar-Poli, L., Freeman, R. E., Spreen, M., Ket, J. C., Vink, A. C., Maratos, A., Crawford, M., Chen, X. J., \& Gold, C. (2017). Music therapy for depression. The Cochrane database of systematic reviews, 11(11), CD004517. https://doi.org/10.1002/14651858.CD004517.pub3

Besson, M., Chobert, J., \& Marie, C. (2011). Transfer of Training between Music and Speech: Common Processing, Attention, and Memory. Frontiers in psychology, 2, 94. https://doi.org/10.3389/fpsyg.2011.00094

Biley, F. (1992). Use of music in therapeutic care. British journal of nursing (Mark Allen Publishing), 1(4), 178179. https://doi.org/10.12968/bjon.1992.1.4.178

Brattico, E., Alluri, V., Bogert, B., Jacobsen, T., Vartiainen, N., Nieminen, S., \& Tervaniemi, M. (2011). A Functional MRI Study of Happy and Sad Emotions in Music with and without Lyrics. Frontiers in psychology, $2,308$. https://doi.org/10.3389/fpsyg.2011.00308

Cai, Y., Pan, X., Zhuangzhong, C.-X., Yinyue de sudu yu diaoshi dui daxuesheng qingxu yingxiang de shizheng yanjiu [An Experimental Research on How Music Excerpts' Tempo and Melody Influence Undergraduates' Emotion]. Psychological Science (China) 2007, 30(1):196-198

Chan, M. F., Wong, Z. Y., \& Thayala, N. (2011). The effectiveness of music listening in reducing depressive symptoms in adults: A systematic review. Complementary Therapies in Medicine, 19(6), 332-348. doi: 10.1016/j.ctim.2011.08.003

Dave Elliott, Remco Polman \& Richard McGregor "Relaxing Music for Anxiety Control", Journal of Music Therapy, 48(3), 2011, 264-288

Frith, S., Straw, W., \&amp; Street, J. (2007). The Cambridge companion to Pop and Rock (pp. 95-105). Cambridge, United Kingdom: Cambridge University Press. 
Garber, J., \& Weersing, V. R. (2010). Comorbidity of Anxiety and Depression in Youth: Implications for Treatment and Prevention. Clinical Psychology: Science and Practice, 17(4), 293-306. doi:10.1111/j.1468-2850.2010.01221.x

Geipel, J., Koenig, J., Hillecke, T. K., Resch, F., \& Kaess, M. (2018). Music-based interventions to reduce internalizing symptoms in children and adolescents: A meta-analysis. Journal of Affective Disorders, 225, 647-656. doi: 10.1016/j.jad.2017.08.035

Goldbeck, L., \& Ellerkamp, T. (2012). A Randomized Controlled Trial of Multimodal Music Therapy for Children with Anxiety Disorders. Journal of Music Therapy, 49(4), 395-413. doi:10.1093/jmt/49.4.395

Gould M.S., King R., Greenwald S., Fisher P., Schwab-Stone M., Kramer R., et al. Psychopathology associated with suicidal ideation and attempts among children and adolescents. Journal of the American Academy of Child and Adolescent Psychiatry. 1998; 37:915-923. [PubMed: 9735611]

Grocke, Denise; Wigram, Tony (2007). Receptive Methods in Music Therapy: Techniques and Clinical Applications for Music Therapy Clinicians, Educators and Students. Jessica Kingsley Publishers. pp. 45-56.

Guétin, S., Portet, F., Picot, M. C., Pommié, C., Messaoudi, M., Djabelkir, L., Olsen, A. L., Cano, M. M., Lecourt, E., \& Touchon, J. (2009). Effect of music therapy on anxiety and depression in patients with Alzheimer's type dementia: randomised, controlled study. Dementia and geriatric cognitive disorders, 28(1), 36-46.

https://doi.org/10.1159/000229024

International Federation of the Phonographic Industry(IFPI). (2018). Music Consumer Insight Report. https://www.ifpi.org/downloads/Music-Consumer-Insight-Report-2018.pdf

Knapp, M., Mccrone, P., Fombonne, E., Beecham, J., \& Wostear, G. (2002). The Maudsley long-term follow-up of child and adolescent depression. British Journal of Psychiatry, 180(1), 19-23. doi:10.1192/bjp.180.1.19

Leubner, D., \& Hinterberger, T. (2017). Reviewing the Effectiveness of Music Interventions in Treating Depression. Frontiers in Psychology, 8. doi: 10.3389/fpsyg.2017.01109

Lin, S.-T., Yang, P., Lai, C.-Y., Su, Y.-Y., Yeh, Y.-C., Huang, M.-F., \& Chen, C.-C. (2010). Mental Health Implications of Music: Insight from Neuroscientific and Clinical Studies. Harvard Review of Psychiatry, 19(1), 34-46. doi: $10.3109 / 10673229.2011 .549769$

Mast, J. F., \& McAndrew, F. T. (2011). Violent lyrics in heavy metal music can increase aggression in males. North American Journal of Psychology, 13(1), 63-64.

Maughan, B., Collishaw, S., \& Stringaris, A. (2013). Depression in childhood and adolescence. Journal of the Canadian Academy of Child and Adolescent Psychiatry = Journal de l'Académie canadienne de psychiatrie de l'enfant et de l'adolescent, 22(1), 35-40.

Miranda, D. (2013). The role of music in adolescent development: much more than the same old song. International Journal of Adolescence and Youth, 18(1), 5-22. doi: 10.1080/02673843.2011.650182 
Morgan, A. J., \& Jorm, A. F. (2008). Self-help interventions for depressive disorders and depressive symptoms: a systematic review. Annals of General Psychiatry, 7(1). doi: 10.1186/1744-859x-7-13

Rohde, P., Lewinsohn, P. M., \& Seeley, J. R. (1994). Are Adolescents Changed by an Episode of Major Depression? Journal of the American Academy of Child \& Adolescent Psychiatry, 33(9), 1289-1298. doi:10.1097/00004583199411000-00010

Sethi, S. (2010). The Use of Computerized Self-Help Packages to Treat Adolescent Depression and Anxiety. Journal of Technology in Human Services, 28(3), 144-160. Retrieved from https://doi.org/10.1080/15228835.2010.508317

Skokauskas, N., Fung, D., Flaherty, L. T., Klitzing, K. V., Pūras, D., Servili, C., . . Guerrero, A. (2019). Shaping the future of child and adolescent psychiatry. Child and Adolescent Psychiatry and Mental Health, 13(1). doi:10.1186/s13034-019-0279-y

Suvi S., Christian G. \& Katrina M., "Development and validation of the Healthy-Unhealthy Music Scale", Child and Adolescent Mental Health 20, No. 4, 2015, pp. 210-217

Thapar, A. K., Collishaw, S. K., Potter, R. K., \& Thapar, A. K. (2010). Managing and preventing depression in adolescents. Bmj, 340(Jan 22), c209-c209. doi: 10.1136/bmj.c209

Vanweelden, K., \& Cevasco, A. M. (2007). Repertoire Recommendations by Music Therapists for Geriatric Clients During Singing Activities. Music Therapy Perspectives, 25(1), 4-12. doi: 10.1093/mtp/25.1.4

World Health Organization. (2019, October 23). Adolescent mental health. Retrieved from https://www.who.int/news-room/fact-sheets/detail/adolescent-mental-health.

Ye, W.-F., Xu, J.-M., (1993). “Zonghexing yiyuan jiaolu yiyu liangbiao"zai zonghe yiyuan bingren zhong de yingyong yu pingjia [Application and Evaluation of " Hospital Anxiety and Depression Scale" in General Hospital Patients]. Chinese Journal of Behavioral Medical and Brain Science, 1993(3):17-19.

Zhong, J., Qian, M.-Y., (2005). Zhongwen qingxu xinrongci jiancebiao de bianzhi yu xinxiaodu yanjiu [A Study of Development and Validation of Chinese Mood Adjective Check List]. Chinese Journal of Clinical Psychology, 13(1), 9-13.

Zigmond, A. S., \& Snaith, R. P. (1983). The Hospital Anxiety and Depression Scale. Acta Psychiatrica Scandinavica, 67(6), 361-370. https://doi.org/10.1111/j.1600-0447.1983.tb09716.x 